Pure and Applied Mathematics Quarterly

Volume 7, Number 4

(Special Issue:

In memory of Eckart Viehweg)

1407-1426, 2011

\title{
On Endomorphisms of Fano Manifolds of Picard Number One
}

\author{
Jun-Muk Hwang* and Noboru Nakayama \\ Dedicated to the memory of Eckart Viehweg
}

\begin{abstract}
Let $X$ be a Fano manifold of Picard number one different from the projective space. It has been conjectured that a surjective endomorphism $X \rightarrow X$ must be bijective. In this article, we will prove a weaker version of the conjecture: a surjective endomorphism $X \rightarrow X$ which is étale outside a completely invariant divisor is bijective. As applications of this result, the conjecture is confirmed in the case where the variety of minimal rational tangents of $X$ is linear and in the case where $X$ is quasi-homogeneous.
\end{abstract}

Keywords: endomorphism of a projective variety, Fano manifold, variety of minimal rational tangents.

\section{INTRODUCTION}

We will work over the complex number field $\mathbb{C}$. It seems that the following has been a folklore since 1980's.

Conjecture 1.1. Let $X$ be a Fano manifold of Picard number one different from the projective space. Then a surjective endomorphism $X \rightarrow X$ must be bijective.

Received: Apr. 1, 2010; Revised: Oct. 31, 2010.

2000 Mathematics Subject Classification. 14J45, 14M17, 32H50

*supported by Basic Science Research Program (ASARC) through NRF funded by MEST (2009-0063180). 
Up to our knowledge, no general strategy to this conjecture has been suggested. Even testing the conjecture for a specifically given Fano manifold of Picard number one is not easy. For that reason, it is worth studying the conjecture with some additional assumptions on $X$. For example, Conjecture 1.1 was proved for homogeneous spaces in [PS], for hypersurfaces of the projective space in [Be] and for Fano manifolds containing a rational curve with trivial normal bundle in [HM03]; the last work solves Conjecture 1.1 in case $\operatorname{dim} X=3$.

On the other hand, since Conjecture 1.1 predicts that all surjective endomorphisms are just automorphisms, it is somewhat artificial and aesthetically repulsive to work on the conjecture with additional assumptions on the endomorphism. Notwithstanding this, we will study Conjecture 1.1 for a special class of endomorphisms in this paper. We say that an endomorphism $f: X \rightarrow X$ is étale outside a completely invariant divisor if there exists a reduced divisor $D \subset X$ such that $f^{-1}(D):=f^{*}(D)_{\text {red }}=D$ and $\left.f\right|_{X \backslash D}: X \backslash D \rightarrow X \backslash D$ is étale. We will prove the following.

Theorem 1.2. Let $X$ be a Fano manifold of Picard number one different from the projective space. If an endomorphism of $X$ is étale outside a completely invariant divisor, it is bijective.

In fact, our result is slightly stronger. See Theorem 2.1 for the precise statement.

What is our excuse for making this special assumption on the endomorphism? We believe that Theorem 1.2 will be useful in attacking Conjecture 1.1. It seems that, for many examples of $X$, the geometry of rational curves on $X$ forces an arbitrary endomorphism $X \rightarrow X$ to be étale outside a completely invariant divisor. To illustrate this idea, we will use Theorem 1.2 to prove the following.

Theorem 1.3. Let $X$ be a Fano manifold of Picard number one different from the projective space. Suppose that the variety of minimal rational tangents of $X$ is linear. Then a surjective endomorphism $X \rightarrow X$ is bijective.

See Section 6 for the meaning of the assumption on the variety of minimal rational tangents. In practice, the only known examples of Fano manifolds of Picard number one whose variety of minimal rational tangents is linear are those having rational curves with trivial normal bundles. For the latter class of Fano 
manifolds, Theorem 1.3 were already proved in [HM03]. In this sense, Theorem 1.3 is a generalization of a result of [HM03]. However, the proof given here is different from that of [HM03] and conceptually simpler. In particular, the calculation involving discriminantal orders, which was the hardest part in [HM03], is not needed here. Moreover, as far as endomorphisms are concerned, Theorem 1.3 has a theoretical advantage which makes it more useful than [HM03]. As an example, we will use Theorem 1.2 and Theorem 1.3 to prove the following, for which the result of [HM03] is not sufficient.

Theorem 1.4. Let $X$ be a Fano manifold of Picard number one different from the projective space. Assume that $X$ is quasi-homogeneous, i.e., the connected component $\operatorname{Aut}_{o}(X)$ of the group of biregular automorphisms of $X$ has an open orbit in $X$. Then a surjective endomorphism $X \rightarrow X$ is bijective.

This verifies Conjecture 1.1 for quasi-homogeneous cases. Note that quasihomogeneous Fano manifolds of Picard number one cover a large class of examples, much larger than the homogeneous cases of $[\mathrm{PS}]$. See, for example, $[\mathrm{Pq}]$ for interesting examples. Even when $\operatorname{Aut}_{o}(X)$ is reductive, this class of Fano manifolds have not yet been classified.

\section{Proof of Theorem 1.2}

In this section, we will prove the following stronger version of Theorem 1.2.

Theorem 2.1. Let $X$ be an $n$-dimensional Fano manifold of Picard number one and $D \subset X$ a reduced divisor. Assume that there exist a non-isomorphic surjective endomorphism $f: X \rightarrow X$ such that $f^{-1}(D)=D$ and $\left.f\right|_{X \backslash D}: X \backslash D \rightarrow$ $X \backslash D$ is étale. Then $X$ is isomorphic to the projective space $\mathbb{P}_{n}$ and $D$ is a simple normal crossing divisor consisting of $n+1$ hyperplanes.

Given a reduced divisor $D$ in a projective manifold $X$, we define the sheaf $\hat{\Omega}_{X}^{1}(\log D)$ as follows. Let $U \subset X$ be a Zariski open subset with $\operatorname{codim}(X \backslash U) \geq 2$ and $D \cap U$ being a smooth divisor. Denote by $\Omega_{U}^{1}(\log (D \cap U))$ the locally free sheaf of germs of logarithmic 1-forms on $U$ with poles only along $U \cap D$. Using the open immersion $j: U \subset X$, we define

$$
\hat{\Omega}_{X}^{1}(\log D):=j_{*} \Omega_{U}^{1}(\log (D \cap U)) .
$$

This is a reflexive coherent sheaf on $X$. 
Proposition 2.2. In the setting of Theorem 2.1, let $A$ be the ample generator of $\operatorname{Pic}(X) \cong \mathbb{Z}$ and let $q>1$ be the integer with $f^{*} A=q A$ in $\operatorname{Pic}(X)$; in particular, $\operatorname{deg} f=q^{n}$. Then the following hold.

(i) $f^{*}\left(c_{1}(A)^{i}\right)=q^{i} c_{1}(A)^{i} \in \mathrm{H}^{2 i}(X, \mathbb{Z})$ and $f_{*}\left(c_{1}(A)^{j}\right)=q^{n-j} c_{1}(A)^{j} \in$ $\mathrm{H}^{2 j}(X, \mathbb{Z})$ for any $0 \leq i, j \leq n$.

(ii) There is a natural isomorphism $f^{*} \hat{\Omega}_{X}^{1}(\log D) \cong \hat{\Omega}_{X}^{1}(\log D)$. In particular, $K_{X}+D=f^{*}\left(K_{X}+D\right)$.

(iii) For any $i>0, c_{i}\left(\hat{\Omega}_{X}^{1}(\log D)\right) c_{1}(A)^{n-i}=0$.

Proof. (i) is direct from the projection formula. (ii) follows from the fact that $f$ is flat, $f^{-1}(D)=D$ and $f$ is étale outside $D$. For (iii), denoting $\hat{\Omega}_{X}^{1}(\log D)$ by $\mathcal{F}$, (ii) gives $f^{*}\left(c_{i}(\mathcal{F})\right)=c_{i}(\mathcal{F})$ for any $i$. Then

$q^{n-i} c_{i}(\mathcal{F}) c_{1}(A)^{n-i}=f^{*} c_{i}(\mathcal{F})\left(f^{*} c_{1}(A)\right)^{n-i}=(\operatorname{deg} f) c_{i}(\mathcal{F}) c_{1}(A)^{n-i}=q^{n} c_{i}(\mathcal{F}) c_{1}(A)^{n-i}$ implies $c_{i}(\mathcal{F}) c_{1}(A)^{n-i}=0$.

Proposition 2.3. In the setting of Proposition 2.2, the sheaf $\hat{\Omega}_{X}^{1}(\log D)$ is semistable with respect to $A$. In fact, $\hat{\Omega}_{X}^{1}(\log D) \cong \mathcal{O}_{X}^{\oplus n}$.

Proof. Suppose it is not semi-stable with respect to $A$. Then, there is a non-zero coherent sheaf $\mathcal{F} \subset \hat{\Omega}^{1}(\log D)$ such that

$$
\mu_{A}(\mathcal{F}):=\frac{c_{1}(\mathcal{F}) c_{1}(A)^{n-1}}{\operatorname{rank} \mathcal{F}}>0 .
$$

Then $\mu_{A}\left(f^{*} \mathcal{F}\right)=q \mu_{A}(\mathcal{F})$ by $f_{*}\left(c_{1}(A)^{n-1}\right)=q c_{1}(A) \in \mathrm{H}^{2}(X, \mathbb{Z})$ and by the projection formula. For the iterated power $f^{k}=f \circ \cdots \circ f(k \geq 1)$ of $f$, we have $\mu_{A}\left(\left(f^{k}\right)^{*} \mathcal{F}\right)=q^{k} \mu_{A}(\mathcal{F})$. Note that the set

$$
\left\{\mu_{A}(\mathcal{F}) \mid 0 \neq \mathcal{F} \subset \hat{\Omega}^{1}(\log D)\right\}
$$

is bounded from above. Since $\left(f^{k}\right)^{*} \mathcal{F} \subset \hat{\Omega}_{X}^{1}(\log D)$ by Proposition 2.2 (ii), we get a contradiction.

Now if a reflexive sheaf $\mathcal{G}$ on a projective manifold $X$ is semi-stable with respect to an ample line bundle $A$, satisfying $c_{1}(\mathcal{G})=0$ and $c_{2}(\mathcal{G}) c_{1}(A)^{n-2}=0$, then $\mathcal{G}$ is locally free and there exists a sequence

$$
0 \subset \mathcal{E}_{1} \subset \mathcal{E}_{2} \subset \cdots \subset \mathcal{E}_{l}=\mathcal{G}
$$


of subbundles such that $\mathcal{E}_{i} / \mathcal{E}_{i-1}$ is a projectively flat vector bundle with $c_{1}\left(\mathcal{E}_{i} / \mathcal{E}_{i-1}\right)=0$ for any $1 \leq i \leq l$ (cf. [Na, IV.4.1]). We can apply it to $\mathcal{G}=\hat{\Omega}_{X}^{1}(\log D)$ by Proposition 2.2 (iii). Then, $\hat{\Omega}_{X}^{1}(\log D)$ is free, since $X$ is simply connected and

$$
\operatorname{dim} \operatorname{Ext}_{X}^{1}\left(\mathcal{O}_{X}, \mathcal{O}_{X}\right)=\operatorname{dim} H^{1}\left(X, \mathcal{O}_{X}\right)=0
$$

The proof of the following result is taken from [NZ, Lemma 5.3 and Proposition $5.4]$.

Proposition 2.4. In the setting of Theorem 2.1, there is an open subset $U \subset X$ such that $D \cap U$ is a normal crossing divisor and $\operatorname{codim}(X \backslash U) \geq 3$.

Proof. Let $\nu: \widetilde{D} \rightarrow D \subset X$ be the normalization of $D$ and $\mathbf{c}$ be the conductor of $D$, regarded as a Weil divisor on $\widetilde{D}$. The adjunction formula gives

$$
K_{\widetilde{D}}+\mathbf{c}=\nu^{*}\left(K_{X}+D\right) .
$$

There is an endomorphism $h: \widetilde{D} \rightarrow \widetilde{D}$ such that $\nu \circ h=f \circ \nu$ and its ramification divisor $R_{h}$ is $h^{*}(\mathbf{c})-\mathbf{c}$. In fact, we have $K_{\widetilde{D}}+\mathbf{c}=h^{*}\left(K_{\widetilde{D}}+\mathbf{c}\right)$ from $K_{X}+D=$ $f^{*}\left(K_{X}+D\right)$ in Proposition 2.2 (ii). Moreover, $\operatorname{deg} h=q^{n-1}$ by $h^{*} \nu^{*}(A)=$ $\nu^{*} f^{*}(A)=q \nu^{*}(A)$, where $q$ is as in Proposition 2.2.

We will show that $\mathbf{c}$ is reduced. Let $\Gamma$ be an irreducible component of $\mathbf{c}$ and $\Theta$ be an irreducible component of $h^{-1}(\Gamma)$. We set $a:=\operatorname{mult}_{\Theta} h^{*}(\Gamma)$. Then

$$
a-1=\operatorname{mult}_{\Theta}\left(R_{h}\right)=\operatorname{mult}_{\Theta}\left(h^{*} \mathbf{c}\right)-\operatorname{mult}_{\Theta}(\mathbf{c})=a \operatorname{mult}_{\Gamma}(\mathbf{c})-\operatorname{mult}_{\Theta}(\mathbf{c}) .
$$

Consequently,

$$
\operatorname{mult}_{\Theta}(\mathbf{c})-1=a\left(\operatorname{mult}_{\Gamma}(\mathbf{c})-1\right) .
$$

Thus, $\Theta$ is contained in c. By considering the number of irreducible components of $\mathbf{c}$, we infer that $\Theta \mapsto h(\Theta)$ induces a permutation of the set of irreducible components of c. In particular, $h^{*}(\Gamma)=a \Theta$. Replacing $h$ by some iteration $h^{m}$, we may assume that $h^{*}(\Gamma)=a \Gamma$. Since $h^{*} \nu^{*}(A) \sim q \nu^{*}(A)$ and $\operatorname{deg} h=q^{n-1}$, we have $a=q$ by

$$
q^{n-1} \Gamma \nu^{*}(A)^{n-2}=h^{*}(\Gamma) h^{*} \nu^{*}(A)^{n-2}=a q^{n-2} \Gamma \nu^{*}(A)^{n-2}>0 .
$$

Thus $h^{*}(\Gamma)=q \Gamma$ and for each positive integer $k,\left(h^{k}\right)^{*}(\Gamma)=q^{k} \Gamma$ for the iterated endomorphism $h^{k}$. Then

$$
\operatorname{mult}_{\Gamma}(\mathbf{c})-1=q^{k}\left(\operatorname{mult}_{\Gamma}(\mathbf{c})-1\right)
$$


by (1). Thus, $\operatorname{mult}_{\Gamma}(\mathbf{c})=1$, proving that $\mathbf{c}$ is reduced.

If a plane curve has a reduced conductor over a singular point, then the singularity is nodal. Hence, $D$ has only normal crossing singularities in codimension one.

Now we are ready to prove Theorem 2.1.

Proof of Theorem 2.1. Let $U$ be the open subset in Proposition 2.4. Since $\operatorname{codim}(X \backslash U) \geq 3$, we have an isomorphism

$$
\mathbb{C} \cong \mathrm{H}^{1}\left(X, \Omega_{X}^{1}\right) \cong \mathrm{H}^{1}\left(U, \Omega_{U}^{1}\right)
$$

On the other hand, we have an exact sequence

$$
\left.0 \longrightarrow \Omega_{U}^{1} \longrightarrow \Omega_{U}^{1}(\log (D \cap U)) \longrightarrow \nu_{*} \mathcal{O}_{\widetilde{D}}\right|_{U} \longrightarrow 0
$$

for the normalization $\nu: \widetilde{D} \rightarrow D$. Here, $\widetilde{D}$ is the disjoint union of the normalizations $\widetilde{D}_{i}$ of irreducible components $D_{i}$ of $D$. Thus, $\operatorname{dim} \mathrm{H}^{0}\left(U, \nu_{*} \mathcal{O}_{\widetilde{D}}\right)$ is the number $l$ of irreducible components of $D$. The connecting homomorphism

$$
\mathrm{H}^{0}\left(U, \nu_{*} \mathcal{O}_{\widetilde{D}}\right) \longrightarrow \mathrm{H}^{1}\left(U, \Omega_{U}^{1}\right) \cong \mathrm{H}^{1}\left(X, \Omega_{X}^{1}\right)
$$

essentially sends a generator 1 of $\mathcal{O}_{\widetilde{D}_{i}}$ for each component $D_{i}$ to the first Chern class $c_{1}\left(D_{i}\right)$. From

$$
\operatorname{dim} H^{0}\left(U, \Omega_{U}^{1}\right)=\operatorname{dim} H^{0}\left(X, \Omega_{X}^{1}\right)=0, \quad \operatorname{dim} H^{1}\left(U, \Omega_{U}^{1}\right)=\operatorname{dim} H^{1}\left(X, \Omega_{X}^{1}\right)=1,
$$

we get

$$
l-1=\operatorname{dim} \mathrm{H}^{0}\left(U, \Omega_{U}^{1}(\log (D \cap U))\right)=\operatorname{dim} \mathrm{H}^{0}\left(X, \hat{\Omega}_{X}^{1}(\log D)\right)=n
$$

where the last equality is from Proposition 2.3. Since $K_{X}+D=0$ by Proposition $2.3,-K_{X}=m A$ for some positive integer $m \geq n+1$. Thus, $X \cong \mathbb{P}_{n}$ by Kobayashi-Ochiai's criterion $[\mathrm{KO}]$. Moreover, $m=l=n+1$ implies that each irreducible component $D_{i}$ is a hyperplane. The normal crossing property of $D=\sum D_{i}$ is verified in [NZ, Proposition 5.6].

\section{Free immersed submanifolds With trivial normal BUndLe}

Let $X$ be a non-singular projective variety. 
Definition 3.1. A finite morphism $\nu: V \rightarrow X$ is called an immersion from a projective manifold if $V$ is a non-singular projective variety with $\operatorname{dim} V<\operatorname{dim} X$ and $\nu$ is unramified and generically injective. If the normal bundle $N_{\nu}=N_{V / X}$ is a trivial bundle of $\operatorname{rank} \operatorname{dim} X-\operatorname{dim} V=\operatorname{codim} \nu(V)>0$ in addition, then $\nu$ is called an immersion with trivial normal bundle. In this case, the image $\nu(V)$ is called an immersed submanifold with trivial normal bundle.

For an immersion $\nu: V \rightarrow X$ from a projective manifold with trivial normal bundle, we have an exact sequence

$$
0 \rightarrow T_{V} \rightarrow \nu^{*} T_{X} \rightarrow N_{V / X} \simeq \mathcal{O}_{V}^{\oplus c} \rightarrow 0
$$

for $c=\operatorname{codim} \nu(V)$. In particular, $K_{V}=\nu^{*} K_{X}$. Therefore, if $X$ is a Fano manifold, then so is $V$. A projective space $\mathbb{P}_{n}$ does not have an immersed submanifold with trivial normal bundle since the tangent bundle is ample.

Definition 3.2. Let $\nu: \mathcal{V} \rightarrow X \times \mathcal{W}$ be a finite morphism for algebraic schemes $\mathcal{V}$ and $\mathcal{W}$. Let $\varphi: \mathcal{V} \rightarrow X$ and $\pi: \mathcal{V} \rightarrow \mathcal{W}$ be the morphisms induced by projections. If $\pi$ is a smooth morphism with connected fibers and $\left.\nu\right|_{V_{w}}=\left.\varphi\right|_{V_{w}}: V_{w}:=\pi^{-1}(w) \rightarrow X$ is an immersion, then $\nu$ is called a family of immersions from projective manifolds parametrized by $\mathcal{W}$

We can consider $\nu$ as a deformation of the holomorphic map $\left.\nu\right|_{V_{w}}$ varying the source and fixing the target. In particular, we have the characteristic map

$$
T_{w}(\mathcal{W}) \rightarrow \mathrm{H}^{0}\left(V_{w}, N_{V_{w} / X}\right)
$$

for the deformation $\mathcal{V} \rightarrow X \times \mathcal{W}$ of the non-degenerate holomorphic map $\left.\nu\right|_{V_{w}}$ in the sense of Horikawa [Ho], where $T_{w}(\mathcal{W})$ denotes the tangent space of $\mathcal{W}$ at $w$. The proof of the following lemma, which is straight forward using basic deformation theory, will be skipped.

Lemma 3.3. Let $\nu: \mathcal{V} \rightarrow X \times \mathcal{W}$ be a finite morphism for algebraic schemes $\mathcal{V}$, $\mathcal{W}$, and let $\varphi: \mathcal{V} \rightarrow X$ and $\pi: \mathcal{V} \rightarrow \mathcal{W}$ be the morphisms induced from projections. Assume that $\pi$ is a smooth morphism with connected fibers. For a given point $w \in \mathcal{W}$ and $V=V_{w}=\pi^{-1}(w)$, the following three conditions are mutually equivalent:

(i) $\left.\nu\right|_{V}: V \rightarrow X$ is an immersion with trivial normal bundle and $\varphi: \mathcal{V} \rightarrow X$ is étale at a point of $V$. 
(ii) $\mathcal{W}$ is non-singular at $w, \nu$ is a family of immersions over an open neighborhood of $w$ in $\mathcal{W},\left.\nu\right|_{V}$ has trivial normal bundle, and the characteristic map $T_{w}(\mathcal{W}) \rightarrow \mathrm{H}^{0}\left(V, N_{V / X}\right)$ is an isomorphism.

(iii) $\nu$ is a family of immersions over an open neighborhood of $w$ in $\mathcal{W}$ and $\varphi$ is étale along $V$.

A family $\mathcal{V} \rightarrow X \times \mathcal{W}$ of immersions from projective manifolds is called étale if the first projection $\mathcal{V} \rightarrow X$ is étale. Let $\nu=(\varphi, \pi): \mathcal{V} \rightarrow X \times \mathcal{W}$ be an étale family of immersions from projective manifolds. Then, $\left.\nu\right|_{V_{w}}=\left.\varphi\right|_{V_{w}}: V_{w}=\pi^{-1}(w) \rightarrow$ $X$ is an immersion with trivial normal bundle for any $w \in \mathcal{W}$ by Lemma 3.3. Moreover, $\mathcal{V} \rightarrow X \times \mathcal{W}$ is a versal family of the deformation of $\left.\nu\right|_{V_{w}}$ for any $w \in \mathcal{W}$ by Lemma 3.3 and [Ho].

We can consider the push-forward $\nu_{*}\left(V_{w}\right)$ as an algebraic cycle of $X$ associated with the subvariety $\nu\left(V_{w}\right)$ for any $w$. Moreover, if $\mathcal{W}$ is normal, then the pushforward $\nu_{*}(\mathcal{V})$ is regarded as a family of algebraic cycles of $X$ parametrized by $\mathcal{W}$. Thus, in this case, we have an associated morphism from $\mathcal{W}$ to the Chow variety $\operatorname{Chow}(X)$ of $X$.

Definition 3.4. A positive-dimensional closed subvariety $M$ of $\operatorname{Chow}(X)$ is called a component of the locus of free immersed submanifolds with trivial normal bundle (FIT, for short) if $M$ is the closure of the image of $\mathcal{W} \rightarrow \operatorname{Chow}(X)$ induced from an étale family $\mathcal{V} \rightarrow X \times \mathcal{W}$ of immersions from projective manifolds. The set of FITs of $X$ is denoted by $\operatorname{FIT}(X)$.

An example of an FIT is provided by the fibers of a surjective morphism $X \rightarrow Y$ with $\operatorname{dim} X>\operatorname{dim} Y$. More interesting examples arise from minimal rational curves on Fano manifolds. As explained in [HM03], if a Fano manifold $X$ contains a rational curve with trivial normal bundle, such a rational curve can be deformed to cover an open subset in $X$. The family of these deformations will give rise to an FIT. Quite often, e.g., when $X$ has Picard number one, this FIT cannot come from a surjective morphism $X \rightarrow Y$. There are many examples of Fano manifolds of Picard number 1 containing rational curves with trivial normal bundles. They provide interesting examples of FIT's. For example, any Fano threefold $X$ of Picard number one, excepting $\mathbb{P}_{3}$ and the quadric hypersurface $X \subset \mathbb{P}_{4}$, admits an FIT, as noted in [HM03, p.628]. More generally, an FIT arises whenever we have a minimal component of the space of rational curves 
on $X$ whose variety of minimal rational tangents is linear, as we will see in Proposition 6.1.

Theorem 3.5. Let $X$ be a Fano manifold. Then $\operatorname{FIT}(X)$ is a finite set.

Proof. We recall the fact that the Fano manifolds of fixed dimension are bounded (cf. [Kr, V.2]). Thus, there exist finitely many smooth families $\Pi_{i}: \mathcal{X}_{i} \rightarrow \mathcal{S}_{i}$ of Fano manifolds such that, for any $M \in \operatorname{FIT}(X)$, there exist an étale family $\nu=$ $(\varphi, \pi): \mathcal{V} \rightarrow X \times \mathcal{W}$ of immersions from projective manifolds defining $M$ as the closure of the image of $\mathcal{W} \rightarrow \operatorname{Chow}(X)$, a morphism $\sigma: \mathcal{W} \rightarrow \mathcal{S}_{i}$ for some $i$, and an isomorphism $\mathcal{V} \simeq \mathcal{X}_{i} \times \mathcal{S}_{i} \mathcal{W}$ over $\mathcal{W}$. We fix $M, \nu: \mathcal{V} \rightarrow X \times \mathcal{W}$, and $\sigma: \mathcal{W} \rightarrow \mathcal{S}_{i}$. We write $\Pi: \mathcal{X} \rightarrow \mathcal{S}$ for $\Pi_{i}: \mathcal{X}_{i} \rightarrow \mathcal{S}_{i}$ for the $i$. The morphism $\nu$ defines a morphism $[\nu]: \mathcal{W} \rightarrow \mathcal{H}$ into the relative Hom scheme $\mathcal{H}:=\operatorname{Hom}_{\mathcal{S}}(\mathcal{X}, X \times \mathcal{S})$ over $\mathcal{S}$. Here, $\sigma=q \circ[\nu]$ for the structure morphism $q: \mathcal{H} \rightarrow \mathcal{S}$. Note that $\mathcal{H}$ is regarded as an open subscheme of the relative Hilbert scheme of $\mathcal{X} \times \mathcal{S}(X \times \mathcal{S}) \simeq \mathcal{X} \times X$ over $\mathcal{S}$. Let

$$
\Lambda: \mathcal{X} \times \mathcal{S} \mathcal{H} \rightarrow(X \times \mathcal{S}) \times{ }_{\mathcal{S}} \mathcal{H}=X \times \mathcal{H}
$$

be the universal family for the Hom scheme $\mathcal{H}$ and let

$$
\Psi=\Pi \times_{\mathcal{S}} \operatorname{id}_{\mathcal{H}}: \mathcal{X} \times_{\mathcal{S}} \mathcal{H} \rightarrow \mathcal{H}
$$

be the second projection. For a point $t \in \mathcal{H}$, let

$$
\Lambda_{t}: \mathcal{X}_{t}:=\Psi^{-1}(t)=\left(\mathcal{X} \times_{\mathcal{S}} \mathcal{H}\right) \times_{\mathcal{H}}\{t\} \simeq \Pi^{-1}(q(t)) \rightarrow(X \times \mathcal{H}) \times_{\mathcal{H}}\{t\}=X
$$

be the base change of $\Lambda$ by $\{t\} \rightarrow \mathcal{H}$.

Claim 3.6. Let $\mathcal{T}$ be the set of points $t \in \mathcal{H}$ such that $\Lambda_{t}$ is an immersion with trivial normal bundle. Then $\mathcal{T}$ is an open subset and $\Lambda: \Psi^{-1}(\mathcal{T}) \rightarrow X \times \mathcal{T}$ is a family of immersions.

Proof. Let $t$ be a point of $\mathcal{T}$. For the proof, we may replace $\mathcal{H}$ with an open neighborhood of $t$ in $\mathcal{H}$. Then $\Lambda$ is a finite morphism over an open neighborhood of $t$ in $\mathcal{H}$. It is easy to see that $\Lambda$ is an immersion over an open neighborhood of $t$ in $\mathcal{H}$. Thus, we may assume that $\Lambda$ is a family of immersions. It is enough to show that $\mathcal{T}$ is an open subset. For the normal bundle

$$
\mathcal{N}:=N_{\Lambda}=N_{\mathcal{X} \times \mathcal{S}} \mathcal{H} X \times \mathcal{H}
$$

and for the fiber $\mathcal{X}_{t}=\Psi^{-1}(t)$, we have an isomorphism $\left.\mathcal{N}\right|_{\mathcal{X}_{t}} \simeq N_{\mathcal{X}_{t} / X}$. Since this is trivial of rank $c:=\operatorname{dim} X-\operatorname{dim} \mathcal{X}_{t}$ and $\mathcal{X}_{t}$ is Fano, $\mathrm{H}^{p}\left(\mathcal{X}_{t}, \mathcal{N} \mid \mathcal{X}_{t}\right)=0$ for 
any $p>0$. Applying the upper semi-continuity theorem and the base change theorem to $\Psi$ and $\mathcal{N}$, we see that $\Psi_{*} \mathcal{N}$ is a locally free sheaf of rank $c$ and

$$
\Psi_{*} \mathcal{N} \otimes \mathbb{C}(t) \simeq \mathrm{H}^{0}\left(\mathcal{X}_{t},\left.\mathcal{N}\right|_{\mathcal{X}_{t}}\right) .
$$

Thus, $\Psi^{*} \Psi_{*} \mathcal{N} \rightarrow \mathcal{N}$ is isomorphic along $\mathcal{X}_{t}$. Therefore, $\Lambda_{t^{\prime}}$ is an immersion with trivial normal bundle for any point $t^{\prime}$ of an open neighborhood of $t$ in $\mathcal{W}$. Thus, $\mathcal{T}$ is open.

Claim 3.7. $\mathcal{T}$ has only finitely many irreducible components.

Proof. We consider the relative ample divisors $-K_{\mathcal{X}}$ on $\mathcal{X}$ and $p_{1}^{*}\left(-K_{X}\right)$ on $X \times \mathcal{S}$ respectively with respect to $\mathcal{S}$, where $p_{1}: X \times \mathcal{S} \rightarrow X$ denotes the first projection. These two divisors define a relative ample divisor on $\mathcal{X} \times X$ over $\mathcal{S}$. Note that $-K_{\mathcal{X}_{t}}=\Lambda_{t}^{*}\left(-K_{X}\right)$ for $t \in \mathcal{T}$. By the boundedness of $\left(-K_{V}\right)^{\operatorname{dim} V}$ for the Fano manifolds $V$, for any $s \in \mathcal{S}$, the open subset $\mathcal{T} \cap q^{-1}(s) \subset \operatorname{Hilb}\left(\Pi^{-1}(s) \times X\right)$ is contained in a union of finitely many projective subvarieties. Thus, the closure $\overline{\mathcal{T}} \subset \operatorname{Hilb}_{\mathcal{S}}(\mathcal{X} \times X)$ is proper over $\mathcal{S}$. Hence, the Claim follows.

Proof of Theorem 3.5 continued. By Claim 3.7, there exist finitely many families of immersions $\nu_{j}=\left(\varphi_{j}, \pi_{j}\right): \mathcal{U}_{j} \rightarrow X \times \mathcal{T}_{j}(j=1,2, \ldots, m)$ from projective manifolds satisfying the following conditions:

- $\mathcal{T}_{j}$ is an irreducible algebraic variety for any $j$.

- $\pi_{j}: \mathcal{U}_{j} \rightarrow \mathcal{T}_{j}$ is a smooth family of Fano manifolds for any $j$.

- The restriction $\left.\nu_{j}\right|_{\mathcal{U}_{t}}: \mathcal{U}_{t} \rightarrow X$ for $\mathcal{U}_{t}:=\pi_{j}^{-1}(t)$ is an immersion with trivial normal bundle for any $j$ and any $t \in \mathcal{T}_{j}$.

- For any $M \in \operatorname{FIT}(X)$, there exist an étale family $\nu: \mathcal{V} \rightarrow X \times \mathcal{W}$ of immersions from projective manifolds and a morphism $\mathcal{W} \rightarrow \mathcal{T}_{j}$ for some $j$ such that $M$ is the closure of the image of $\mathcal{W} \rightarrow \operatorname{Chow}(X)$ and such that $\nu: \mathcal{V} \rightarrow X \times \mathcal{W}$ is just the pullback of $\nu_{j}$ by $\mathcal{W} \rightarrow \mathcal{T}_{j}$.

For a given $M \in \operatorname{FIT}(X)$, let $\nu=(\varphi, \pi): \mathcal{V} \rightarrow X \times \mathcal{W}$ and $\mathcal{W} \rightarrow \mathcal{T}_{j}$ be as above. We shall show that $M$ is just the closure of the image of the morphism $\mathcal{T}_{j} \rightarrow \operatorname{Chow}(X)$ associated with the family $\nu_{j}$ of immersions. If this is proved, then the finiteness of $\operatorname{FIT}(X)$ follows. Let $t \in \mathcal{T}_{j}$ be the image of $w \in \mathcal{W}$ by $\mathcal{W} \rightarrow \mathcal{T}_{j}$ and let $V$ be the fiber $\pi^{-1}(w)=\pi_{j}^{-1}(t)$. Now, $\nu: \mathcal{V} \rightarrow X \times \mathcal{W}$ is a versal family of the deformation of the immersion $\left.\varphi\right|_{V}: V \rightarrow X$. Thus, $\mathcal{U}_{j} \rightarrow \mathcal{T}_{j}$ is isomorphic to the pullback of the versal family on an analytic open neighborhood 
$\mathcal{T}_{j}^{\sharp}$ of $t$ in $\mathcal{T}_{j}$. Hence, the image of $\mathcal{T}_{j}^{\sharp} \rightarrow$ Chow $(X)$ is contained in the image of $\mathcal{W} \rightarrow \operatorname{Chow}(X)$. Therefore, the closure of the image of $\mathcal{T}_{j} \rightarrow \operatorname{Chow}(X)$ is $M$. Thus, we are done.

\section{Divisors univalent With Respect to an FIT}

We will use the notation of the previous section. Let $M$ be an FIT of $X$ and let $\mathcal{V} \rightarrow X \times \mathcal{W}$ be an étale family of immersions from projective manifolds defining $M$. Let $Z \rightarrow M$ be the normalization. Then $\mathcal{W} \rightarrow M \subset \operatorname{Chow}(X)$ factors through $Z$. There is a family $\mathcal{Y}$ of algebraic cycles of $X$ parametrized by $Z$. For a point $w \in \mathcal{W}$ and its image $z$ in $Z, \mathcal{Y} \cap(X \times\{z\})$ coincides with the cycle $\nu_{*}\left(\mathcal{V}_{w}\right)$. Let $Y$ be the normalization of the irreducible component of $\operatorname{Supp}(\mathcal{Y})$ which dominates $Z$. Then we have a finite birational morphism $\mathcal{V} \rightarrow Y{ }{ }_{Z} \mathcal{W}$. In particular, $\mathcal{V} \rightarrow X \times \mathcal{W}$ is determined by the morphism $\mathcal{W} \rightarrow Z$. Let $Y \rightarrow X \times Z$ be the induced generically injective morphism. Let $\mu: Y \rightarrow X$ and $\rho: Y \rightarrow Z$ be the morphisms induced from the projections. We define $Z_{o} \subset Z$ to be the maximum open subset such that $\rho: Y_{o} \rightarrow Z_{o}$ is smooth and $\mu: Y_{o} \rightarrow X$ is étale for $Y_{o}:=\rho^{-1}\left(Z_{o}\right)$. Then $Z_{o}$ is a non-singular dense Zariski open subset of $Z$ and $Y_{O} \rightarrow X \times Z_{o}$ is an étale family of immersions.

Definition 4.1. The morphism $(\mu, \rho): Y \rightarrow X \times Z$ above is called the normalized realization of $M$. The étale family $Y_{o} \rightarrow X \times Z_{o}$ of immersions is called the smooth realization of $M$. A smooth member of $M$ means the immersion $\mu: \rho^{-1}(z) \rightarrow X$ with trivial normal bundle for a point $z \in Z_{o}$.

Lemma 4.2. Let $X$ be a Fano manifold of Picard number one. Let $(\mu, \rho): Y \rightarrow$ $X \times Z$ be the normalized realization of an FIT of $X$. Then $\operatorname{deg} \mu>1$.

Proof. Assume that $\mu$ is birational. Then $\mu: Y_{o} \rightarrow X$ is an open immersion. For a non-zero effective Cartier divisor $\Theta$ of $Z$, we have

$$
\mu^{*} \mu_{*} \rho^{*}(\Theta)=\rho^{*}(\Theta)+E
$$

for an effective divisor $E$ supported on $Y \backslash Y_{o}$. The non-zero effective divisor $\mu_{*}\left(\rho^{*}(\Theta)\right)$ is not ample since it does not intersect $\mu\left(\rho^{-1}(z)\right)$ for a point $z \in Z_{o} \backslash \Theta$. This is a contradiction. Thus, $\operatorname{deg} \mu>1$.

Definition 4.3. Let $M$ be an FIT of $X$ and let $(\mu, \rho): Y \rightarrow X \times Z$ be the normalized realization of $M$. 
(i) For a prime divisor $H$ of $X$, let $\mu^{*}(H)=H^{h}+H^{v}$ be the decomposition into the horizontal part $H^{h}$ and the vertical part $H^{v}$ with respect to $\rho: Y \rightarrow Z$. Then, $\rho\left(\operatorname{Supp} H^{v}\right) \neq Z$ and any irreducible component of $H^{h}$ dominates $Z$.

(ii) A prime divisor $H$ is said to be univalent with respect to $M$ if $H^{h}$ is irreducible and $\mu$ induces a birational morphism $H^{h} \rightarrow H$.

Lemma 4.4. Let $H$ be a univalent prime divisor with respect to an FIT M. Then, for a general point $x \in H$, there exists a unique smooth member $\nu: V \rightarrow X$ of $M$ such that $x \in \nu(V)$ and $\nu(V) \not \subset H$. Moreover, the image $\nu(V)$ is non-singular at $x$.

Proof. Let $(\mu, \rho): Y \rightarrow X \times Z$ be the normalized realization of $M$. Let $D$ be an irreducible component of $H^{v}$ such that $D \cap Y_{o} \neq \emptyset$. Then $D \cap Y_{o}=\rho^{-1}\left(\rho(D) \cap Z_{o}\right)$ since $\rho$ is smooth over $Z_{o}$. Therefore,

$$
\left.\mu^{*}(H)\right|_{Y_{o}}=\left.H^{h}\right|_{Y_{o}}+\rho^{*}(\Theta)
$$

for an effective divisor $\Theta$ on $Z_{o}$. Let $x \in H \cap \mu\left(Y_{o}\right)$ be a general point. Then $\mu^{-1}(x) \cap H^{h}=\{y\}$ for a unique point $y \in Y_{o}$. If $y^{\prime} \in \mu^{-1}(x) \backslash\{y\}$, then $y^{\prime} \in \rho^{-1}(\Theta), \rho^{-1}\left(\rho\left(y^{\prime}\right)\right) \subset H^{v}$ and $\mu\left(\rho^{-1}\left(\rho\left(y^{\prime}\right)\right)\right) \subset H$. Thus, $\rho^{-1}(\rho(y)) \rightarrow X$ is the unique smooth member $\nu: V \rightarrow X$ of $M$ with $x \in \nu(V)$ and $\nu(V) \not \subset H$. Since $\rho^{-1}(y) \cap \mu^{-1}(x)=\{y\}, x$ is a non-singular point of $\nu(V)=\mu\left(\rho^{-1}(\rho(y))\right)$.

Let $f: \tilde{X} \rightarrow X$ be a generically finite surjective morphism from another projective manifold $\tilde{X}$. We consider pulling back of FITs of $X$ to $\tilde{X}$. Let $M$ be an FIT of $X$ and $(\mu, \rho): Y \rightarrow X \times Z$ the normalized realization of $M$. Let $\tilde{Y}$ be the normalization of an irreducible component of the fiber product $\tilde{X} \times_{X} Y$ which dominates $Y$ and let $\tilde{\mu}: \tilde{Y} \rightarrow \tilde{X}$ and $f_{Y}: \tilde{Y} \rightarrow Y$ be the induced morphisms. As the Stein factorization of the composition $\rho \circ f_{Y}: \tilde{Y} \rightarrow Y \rightarrow Z$, we have a proper surjective morphism $\tilde{\rho}: \tilde{Y} \rightarrow \tilde{Z}$ to a normal projective variety $\tilde{Z}$ with connected fibers and a finite morphism $f_{Z}: \tilde{Z} \rightarrow Z$ such that $f_{Z} \circ \tilde{\rho}=\rho \circ f_{Y}$.

Lemma 4.5. In this situation, let $Z_{b} \subset Z_{o}$ be the maximum open subset over which $\rho \circ f_{Y}: \tilde{Y} \rightarrow Z$ is smooth. Set $Y_{b}=\rho^{-1}\left(Z_{b}\right), \tilde{Z}_{b}=f_{Z}^{-1}\left(Z_{b}\right)$, and $\tilde{Y}_{b}=f_{Y}^{-1}\left(Y_{b}\right)$. Then $(\tilde{\mu}, \tilde{\rho}): \tilde{Y}_{b} \rightarrow \tilde{X} \times \tilde{Z}_{b}$ is an étale family of immersions from projective manifolds and the associated morphism $\tilde{Z}_{b} \rightarrow \operatorname{Chow}(\tilde{X})$ is generically injective. Let $\tilde{M}$ be the FIT of $\tilde{X}$ defined by $\tilde{Y}_{b} \rightarrow \tilde{X} \times \tilde{Z}_{b}$. If $f$ is a finite morphism, then $(\tilde{\mu}, \tilde{\rho}): \tilde{Y} \rightarrow \tilde{X} \times \tilde{Z}$ is the normalized realization of $\tilde{M}$. 
Proof. The first assertion can be checked easily using base change, which will be left to the reader. For the second assertion, we have a morphism $j: \tilde{Z}_{b} \rightarrow \bar{Z}$ for the normalization $\bar{Z}$ of $\tilde{M}$. It is enough to prove that $j$ extends to an isomorphism $\tilde{Z} \rightarrow \bar{Z}$ provided that $f$ is finite. Since $f$ is finite, considering the push-forward $f_{*}$ of cycles, we have a finite morphism $f_{*}: \bar{Z} \rightarrow Z$ such that $f_{*} \circ j$ coincides with the composition of $f_{Z}: \tilde{Z}_{b} \rightarrow Z_{b}$ and $Z_{b} \subset Z$. Thus, $j$ extends to a finite morphism $\tilde{Z} \rightarrow \bar{Z}$ since $\tilde{Z} \rightarrow Z$ is also a finite morphism. Hence, $\tilde{Z} \simeq \bar{Z}$ since it is a birational morphism of normal projective varieties. Thus, we are done.

Let $f: \tilde{X} \rightarrow X$ be a generically finite surjective morphism between non-singular projective varieties. Let $\tilde{X} \rightarrow \bar{X} \rightarrow X$ be the Stein factorization. The branch locus $B$ of the finite morphism $\bar{X} \rightarrow X$ is purely of codimension one. We call $B$ the branch divisor of $f$.

Lemma 4.6. Let $f: \tilde{X} \rightarrow X, M,(\mu, \rho): Y \rightarrow X \times Z$ be the same as before. For the branch divisor $B$ of $f$, assume that the horizontal part $B^{h}$ for $B$ with respect to $\rho: Y \rightarrow Z$, in the sense of Definition 4.3, is not zero. Then there is an irreducible component of $\tilde{X} \times_{X} Y$ such that, for the normalization $\tilde{Y}$ of the component and for the induced morphisms $f_{Y}: \tilde{Y} \rightarrow Y, f_{Z}: \tilde{Z} \rightarrow Z$ as above, the inequality $\operatorname{deg}(\tilde{Y} / Y)>\operatorname{deg}(\tilde{Z} / Z)$ holds.

Proof. Assume that $\operatorname{deg}(\tilde{Y} / Y)=\operatorname{deg}(\tilde{Z} / Z)$ for any $\tilde{Y}$. Then

$$
\tilde{Y}_{b} \simeq Y_{b} \times_{Z_{b}} \tilde{Z}_{b}
$$

since everything is smooth over $Z_{b}$. Thus, $f_{Y}: \tilde{Y}_{b} \rightarrow Y_{b}$ is étale over $\rho^{-1}\left(Z_{b}^{\prime}\right)$ for a non-empty Zariski open subset $Z_{b}^{\prime} \subset Z_{b}$. Since it holds for any $\tilde{Y}$, the divisor $\mu^{-1}(B) \cap Y_{b}$ does not dominate $Z_{b}$. This is a contradiction to the assumption: $B^{h} \neq 0$.

Proposition 4.7. Let $X$ be a Fano manifold of Picard number one. For a given $M \in \operatorname{FIT}(X)$, there exist at most finitely many univalent prime divisors of $X$ with respect to $M$.

Proof. Let $(\mu, \rho): Y \rightarrow X \times Z$ be the normalized realization of $M$. We apply Lemmata 4.5 and 4.6 to the generically finite morphism $\mu=f: Y=\tilde{X} \rightarrow X$. Let $\tilde{Y} \rightarrow \tilde{X} \times \tilde{Z}$ be the same as in Lemma 4.6. Also let the symbol $b$ be as in Lemma 4.5. Let $U$ be the open subset $X \backslash \mu\left(Y \backslash Y_{b}\right)$. Then $\mu^{-1}(U) \subset Y_{b}$. It is enough to show that $U \cap H=\emptyset$ for any univalent divisor $H$ of $X$ with respect 
to $M$. Assume the contrary. Since $\mu^{*}(H)=H^{h}+H^{v}$ and $\operatorname{deg} \mu>1$, there is an irreducible component $D \subset H^{v}$ such that $\mu(D)=H$ and $D \cap Y_{b} \neq \emptyset$. Then $D \cap Y_{b}=\rho^{-1}\left(\Theta \cap Z_{b}\right)$ for the prime divisor $\Theta=\rho(D)$ since $\rho$ is smooth over $Z_{b}$. There is an irreducible component $\Gamma_{b}$ of $f_{Y}^{*}\left(D \cap Y_{b}\right)$ such that the closure of $\tilde{\mu}\left(\Gamma_{b}\right)$ is $H^{h}$ for the induced morphism $\tilde{\mu}: \tilde{Y} \rightarrow \tilde{X}$. Then $\Gamma_{b} \rightarrow D \cap Y_{b}$ is a non-birational generically finite morphism by Lemma 4.6. On the other hand, $\Gamma_{b} \rightarrow D \cap Y_{b}$ is birational, since

$$
\Gamma_{b} \subset H^{h} \times_{X}\left(D \cap Y_{b}\right) \subset \tilde{X} \times_{X} Y_{b}
$$

and $H^{h} \rightarrow H$ is birational. This is a contradiction. Hence, any univalent divisor with respect to $M$ is an irreducible component of $X \backslash U$. Thus, we are done.

\section{WEBS AND TANGENTIALLY SPECIAL DIVISORS}

Let $X$ be a non-singular projective variety.

Definition 5.1. Let $M$ be an FIT and let $\nu=(\mu, \rho): Y_{o} \rightarrow X \times Z_{o}$ be the smooth realization of $M$. We call $M$ a web if the following two conditions are satisfied for an open dense subset $U_{M} \subset X$ :

(i) For any $y \in \mu^{-1}\left(U_{M}\right) \cap Y_{o}, \mu(y)$ is a non-singular point of $\mu\left(\rho^{-1}(\rho(y))\right.$.

(ii) For any point $\left(y_{1}, y_{2}\right) \in\left(Y_{o} \times_{X} Y_{o}\right) \backslash\left(Y \times_{Z} Y\right)$ with $x=\mu\left(y_{1}\right)=\mu\left(y_{2}\right) \in$ $U_{M}$

$$
\mu_{*} T_{y_{1}}\left(V_{1}\right) \cap \mu_{*} T_{y_{2}}\left(V_{2}\right)=0 \quad \text { in } \quad T_{x}(X),
$$

where $V_{i}=\rho^{-1}\left(\rho\left(y_{i}\right)\right)$ for $i=1,2$.

Remark 5.2. The condition (i) of Definition 5.1 is equivalent to that the projection $\nu\left(Y_{o}\right) \cap\left(U_{M} \times Z_{o}\right) \rightarrow Z_{o}$ is smooth for the image $\nu\left(Y_{o}\right)$. The condition (ii) of Definition 5.1 is equivalent to that the composition

$$
\left.\left.T_{Y_{o} \times Y_{o} / Z_{o} \times Z_{o}}\right|_{Y_{o} \times X Y_{o}} \rightarrow T_{Y_{o} \times Y_{o}}\right|_{Y_{o} \times{ }_{X} Y_{o}} \rightarrow N_{Y_{o} \times X} Y_{o} / Y_{o} \times Y_{o}
$$

of natural homomorphisms is injective and has maximal rank at any point $\left(y_{1}, y_{2}\right) \in\left(Y_{o} \times_{X} Y_{o}\right) \backslash\left(Y \times_{Z} Y\right)$ with $x=\mu\left(y_{1}\right)=\mu\left(y_{2}\right) \in U_{M}$. Indeed, the fiber of the homomorphism (4) at $\left(y_{1}, y_{2}\right) \in\left(Y_{o} \times_{X} Y_{o}\right) \backslash\left(Y \times_{Z} Y\right)$ is expressed as

$$
T_{y_{1}}\left(V_{1}\right) \oplus T_{y_{2}}\left(V_{2}\right) \ni\left(v_{1}, v_{2}\right) \mapsto \mu_{*}\left(v_{1}\right)-\mu_{*}\left(v_{2}\right) \in T_{x}(X),
$$

where $x=\mu\left(y_{1}\right)=\mu\left(y_{2}\right) \in X$ and $V_{i}=\rho^{-1}\left(\rho\left(y_{i}\right)\right)$ for $i=1,2$. This is because $\mu_{*}: T_{y_{i}}(Y) \rightarrow T_{x}(X)$ is an isomorphism for $i=1,2$ and the tangent space 
$T_{(x, x)}\left(\Delta_{X}\right)$ of the diagonal locus $\Delta_{X} \subset X \times X$ at $(x, x)$ is just the diagonal locus of $T_{x}(X) \oplus T_{x}(X)=T_{(x, x)}(X \times X)$.

Definition 5.3. Let $M$ be a web and let $(\mu, \rho): Y \rightarrow X \times Z$ be the normalized realization of $M$. A prime divisor $H$ of $X$ is called tangentially special if $H^{h} \neq$ $\emptyset$ (cf. Definition 4.3) and there is a coherent subsheaf $\mathcal{L}$ of $\left.T_{X}\right|_{H}$ of rank one satisfying the following conditions:

(i) $\mathcal{L} \cap T_{H}=0$ for the subsheaf $\left.T_{H} \subset T_{X}\right|_{H}$.

(ii) $\mu_{H}^{*} \mathcal{L} \cap\left(\left.T_{Y_{o} / Z_{o}}\right|_{H_{o}^{h}}\right)$ is of rank one on $H_{o}^{h}:=H^{h} \cap Y_{o}$ for the restriction $\mu_{H}: H_{o}^{h} \rightarrow H$ of $\mu$ and for the subsheaf

$$
\left.\left.\left.T_{Y_{o} / Z_{o}}\right|_{H_{o}^{h}} \subset T_{Y_{o}}\right|_{H_{o}^{h}} \simeq \mu^{*}\left(T_{X}\right)\right|_{H_{o}^{h}} \simeq \mu_{H}^{*}\left(\left.T_{X}\right|_{H}\right) .
$$

Remark. In the situation of Definition 5.3, the injection $\left.\mathcal{L} \oplus T_{H} \subset T_{X}\right|_{H}$ is an isomorphism over an open dense subset of $H$. Thus, $\mathcal{L} \otimes \mathbb{C}(x)=\mathcal{L}_{x} \otimes_{\mathcal{O}_{H, x}} \mathbb{C}(x)$ is a one-dimensional subspace of $T_{x}(X)$ for general $x \in X$.

Lemma 5.4. For a web $M$ and for the open subset $U_{M}$ in Definition 5.1, if $H$ is a tangentially special prime divisor with respect to $M$ with $U_{M} \cap H \neq \emptyset$, then $H$ is univalent with respect to $M$.

Proof. Let $H$ be a tangentially special prime divisor with respect to $M$. Let $\mathcal{L}$ be the subsheaf of $\left.T_{X}\right|_{H}$ as in Definition 5.3. For a smooth point $x \in H \cap U_{M}$, assume that the image of $\mathcal{L} \otimes \mathbb{C}(x)$ is a one-dimensional subspace of $T_{x}(X)$ and that $x=\mu\left(y_{1}\right)=\mu\left(y_{2}\right)$ for two points $y_{1}, y_{2} \in H_{o}^{h}$. For $i=1,2$, let $v_{i}$ be a non-zero element of

$$
\left(\mu_{H}^{*} \mathcal{L} \cap\left(\left.T_{Y_{o} / Z_{o}}\right|_{H_{o}^{h}}\right)\right) \otimes \mathbb{C}\left(y_{i}\right) \subset T_{Y_{o} / Z_{o}} \otimes \mathbb{C}\left(y_{i}\right)=T_{y_{i}}\left(V_{i}\right),
$$

where $V_{i}:=\rho^{-1}\left(\rho\left(y_{i}\right)\right)$. Then the images $\mu_{*}\left(v_{1}\right)$ and $\mu_{*}\left(v_{2}\right)$ in $T_{x}(X)$ are non-zero elements contained in $\mathcal{L} \otimes \mathbb{C}(x)$. Therefore, the equality (3) is not satisfied. Hence, $\rho\left(y_{1}\right)=\rho\left(y_{2}\right)$. Thus, $\mu\left(V_{1}\right)=\mu\left(V_{2}\right)$ is singular at $x$. This is a contradiction to the condition (i) of Definition 5.1. Therefore, $H^{h} \rightarrow H$ is birational.

Corollary 5.5. If $X$ is a Fano manifold of Picard number one, then, for a given web, there exist at most finitely many tangentially special prime divisors.

Proof. A tangentially special divisor is an irreducible component of $X \backslash U_{M}$ or univalent with respect to $M$ by Lemma 5.4. Thus, the assertion follows from Proposition 4.7. 
Lemma 5.6. Let $M$ be a web of $X$. Let $f: \tilde{X} \rightarrow X$ be a finite surjective morphism from another non-singular projective variety $\tilde{X}$. Let $\tilde{M}$ be an FIT of $\tilde{X}$ arising from $M$ and $f$ as in Lemma 4.5. Then $\tilde{M}$ is a web.

Proof. Let $U_{\tilde{M}}$ be the open subset $f^{-1}\left(U_{M} \backslash B\right)$ for the branch divisor $B$ of $f$. Then the condition (i) of Definition 5.1 is satisfied for $\tilde{M}$ and $U_{\tilde{M}}$, since $f^{-1}(X \backslash B) \rightarrow X \backslash B$ is étale. Let $(\tilde{\mu}, \tilde{\rho}): \tilde{Y}_{o} \rightarrow \tilde{X} \times \tilde{Z}_{o}$ be the smooth realization of $\tilde{M}$. For $\left(\tilde{y}_{1}, \tilde{y}_{2}\right) \in\left(\tilde{Y}_{o} \times_{\tilde{X}} \tilde{Y}_{o}\right) \backslash\left(\tilde{Y} \times_{\tilde{Z}} \tilde{Y}\right)$ with $\tilde{x}=\tilde{\mu}\left(\tilde{y}_{1}\right)=\tilde{\mu}\left(\tilde{y}_{2}\right) \in U_{\tilde{M}}$, if we set $y_{1}=f_{Y}\left(\tilde{y}_{1}\right)$ and $y_{2}=f_{Y}\left(\tilde{y}_{2}\right)$, then $\left(y_{1}, y_{2}\right) \in\left(Y_{o} \times_{X} Y_{o}\right) \backslash\left(Y \times_{Z} Y\right)$ with $x=f(\tilde{x})=\mu\left(y_{1}\right)=\mu\left(y_{2}\right) \in U_{M}$. Since $f$ is unramified at $\tilde{x},(3)$ implies that

$$
\tilde{\mu}_{*} T_{\tilde{y}_{1}}\left(\tilde{V}_{1}\right) \cap \tilde{\mu}_{*} T_{\tilde{y}_{2}}\left(\tilde{V}_{2}\right)=0 \quad \text { in } \quad T_{\tilde{x}}(\tilde{X})
$$

where $\tilde{V}_{i}=\tilde{\rho}^{-1}\left(\tilde{\rho}\left(\tilde{y}_{i}\right)\right)$ for $i=1,2$. Thus, the condition (ii) of Definition 5.1 is also satisfied for $\tilde{M}$.

Lemma 5.7. In the situation of Lemma 5.6, let $\tilde{H}$ be a prime divisor of $\tilde{X}$ such that $H^{h} \neq 0$ for $H:=f(\tilde{H})$. Either if $f$ is ramified along $\tilde{H}$ or if $f(\tilde{H})$ is tangentially special with respect to $M$, then $\tilde{H}$ is tangentially special with respect to any web $\tilde{M}$ obtained as in Lemma 5.6.

Proof. The horizontal part $\tilde{H}^{h}$ with respect to $\tilde{\rho}$ is not zero for the normalized realization $(\tilde{\mu}, \tilde{\rho}): \tilde{Y} \rightarrow \tilde{X} \times \tilde{Z}$ of $\tilde{M}$.

Assume that $f$ is ramified along $\tilde{H}$. The kernel $\widetilde{\mathcal{L}}$ of

$$
\left.\left.T_{\tilde{X}}\right|_{\tilde{H}} \rightarrow f^{*} T_{X}\right|_{\tilde{H}}
$$

is of rank one and $\widetilde{\mathcal{L}} \cap T_{\tilde{H}}=0$. Let $\tilde{H}_{b}^{h}$ be the open subset $\tilde{H}^{h} \cap \tilde{Y}_{b}$. Then

$$
\left.\operatorname{Ker}\left(\left.\left.T_{\tilde{Y} / \tilde{Z}}\right|_{\tilde{H}^{h}} \rightarrow f_{Y}^{*} T_{Y / Z}\right|_{\tilde{H}^{h}}\right)\right|_{\tilde{H}_{b}^{h}}=\left.\operatorname{Ker}\left(\left.\left.T_{\tilde{Y}}\right|_{\tilde{H}^{h}} \rightarrow f_{Y}^{*} T_{Y}\right|_{\tilde{H}^{h}}\right)\right|_{\tilde{H}_{b}^{h}} \simeq \tilde{\mu}_{\tilde{H}}^{*} \widetilde{\mathcal{L}}
$$

for the morphism $\tilde{\mu}_{\tilde{H}}: \tilde{H}_{b}^{h} \rightarrow \tilde{H}$ induced from $\tilde{\mu}$, since $f_{Z}: \tilde{Z}_{b} \rightarrow Z_{b}$ and $\tilde{\mu}: \tilde{Y}_{b} \rightarrow$ $\tilde{Y}$ are étale. Thus, $\tilde{H}$ is tangentially special with respect to $\tilde{M}$.

Next assume that $H$ is tangentially special with respect to $M$ and that $f$ is not ramified along $\tilde{H}$. Let $\tilde{U} \subset \tilde{X}$ be an open subset such that $f: \tilde{U} \rightarrow X$ is étale and $\tilde{U} \cap \tilde{H} \neq \emptyset$. Let $\mathcal{L}$ be the subsheaf of $\left.T_{X}\right|_{H}$ in Definition 5.3. For the induced morphism $f_{H}: \tilde{H} \rightarrow H$, we set

$$
\widetilde{\mathcal{L}}:=\left.\left.f_{H}^{*} \mathcal{L} \cap T_{\tilde{X}}\right|_{\tilde{H}} \subset f^{*} T_{X}\right|_{\tilde{H}}=f_{H}^{*}\left(\left.T_{X}\right|_{H}\right) .
$$


Then $\widetilde{\mathcal{L}} \cap T_{\tilde{H}}=0$ and $\widetilde{\mathcal{L}}$ is of rank one, since $T_{\tilde{X}} \rightarrow f^{*} T_{X}$ is isomorphic over $\tilde{U}$. Since $\tilde{Y}_{b} \rightarrow Y_{b}$ is étale along $\tilde{\mu}^{-1}(\tilde{U})$, the condition (ii) of Definition 5.3 also holds for $\widetilde{\mathcal{L}}$ and $\tilde{Y} \rightarrow \tilde{X} \times \tilde{Z}$. Thus, we are done.

Theorem 5.8. Let $X$ be a Fano manifold of Picard number one admitting a web. Then any surjective endomorphism $X \rightarrow X$ is bijective.

Proof. For a web $M$ of $X$, let $E_{M}$ be the union of tangentially special prime divisors with respect to $M$. Then $E_{M}$ is a divisor by Corollary 5.5. The set of webs of $X$ is a finite set by Proposition 4.7. Thus, the union $E$ of $E_{M}$ for all the webs $M$ of $X$ is also a divisor. Suppose that there is a surjective endomorphism $f: X \rightarrow X$ of degree $>1$. Then any irreducible component of the ramification divisor of $f$ is contained in $E$ and $f^{-1}(E) \subset E$ by Lemma 5.7. Thus, $f^{-1}(E)=E$ and $f: X \backslash E \rightarrow X \backslash E$ is étale. Then $X$ is a projective space by Theorem 1.2. This contradicts that $X$ has a web.

\section{Proofs of Theorems 1.3 And 1.4}

Let $X$ be a Fano manifold of Picard number one. An irreducible component $\mathcal{K}$ of the space $\operatorname{RatCurves}(X)$ of rational curves (in the sense of $[\mathrm{Kr}]$ ) on $X$ is called a minimal component if for a general point $x \in X$, the subscheme $\mathcal{K}_{x}$ of $\mathcal{K}$ consisting of members passing through $x$ is non-empty and complete. In this case, the subvariety $\mathcal{C}_{x}$ of the projectivized tangent space $\mathbb{P} T_{x}(X)$ consisting of the tangent directions at $x$ of members of $\mathcal{K}_{x}$ is called the variety of minimal rational tangents at $x$ (see [HM04] for more details). We say that the variety of minimal rational tangents of $X$ is linear if $\mathcal{C}_{x}$ is a union of linear subspaces of $\mathbb{P} T_{x}(X)$ for a general $x \in X$. This includes the case when $\mathcal{C}_{x}$ is a finite set. Then we have the following results from [Hw, Propositions 2.1 and 2.2].

Proposition 6.1. Let $X$ be a Fano manifold of Picard number one different from the projective space. Suppose that the variety of minimimal tangents of $X$ is linear of dimension $p \geq 0$. Then $X$ has a web $M$ such that the projection $\rho: Y_{o} \rightarrow Z_{o}$ is a $\mathbb{P}_{p+1}$-bundle for the smooth realization $(\mu, \rho): Y_{o} \rightarrow X \times Z_{o}$ of $M$.

Proof of Theorem 1.3. Under the assumption of Theorem 1.3, $X$ is a Fano manifold of Picard number one different from the projective space and the variety of minimal rational tangents of $X$ is linear of dimension $p \geq 0$ for some 
$p<\operatorname{dim} X-1$. By Proposition $6.1, X$ has a web. Applying Theorem 5.8, any surjective endomorphism $X \rightarrow X$ is bijective, which is the assertion of Theorem 1.3.

We recall the following result from [HM04, p. 62, Corollary 2].

Proposition 6.2. Let $f: X^{\prime} \rightarrow X$ be a surjective generically finite morphism from a projective manifold $X^{\prime}$ to a Fano manifold $X$ of Picard number one. Assume that the variety of minimal rational tangents of $X$ is not linear. Then any holomorphic vector field on $X^{\prime}$ descends to a holomorphic vector field on $X$ such that $f$ is equivariant with respect to the 1-parameter groups of automorphisms of $X^{\prime}$ and $X$ generated by the holomorphic vector fields.

Combining Theorem 1.3 and Proposition 6.2, we have the following.

Proposition 6.3. Let $X$ be a Fano manifold of Picard number one different from the projective space. Let $f: X \rightarrow X$ be a surjective endomorphism. Then $f$ is equivariant with respect to $\operatorname{Aut}_{o}(X)$, in the sense that it induces a homomorphism $\Phi: \operatorname{Aut}_{o}(X) \rightarrow \operatorname{Aut}_{o}(X)$ such that $f(\sigma x)=\Phi(\sigma) f(x)$ for $\sigma \in \operatorname{Aut}_{o}(X)$ and $x \in X$.

Proof. If the variety of minimal rational tangents of $X$ is linear, $f$ is biregular by Theorem 1.3. Otherwise, we apply Proposition 6.2 to get the equivariance.

A projective manifold $X$ is quasi-homogeneous if $\operatorname{Aut}_{o}(X)$ has an open orbit $X_{o} \subset X$. The complement $E$ of $X_{o}$ is called the boundary of $X$. The boundary $E \subset X$ is a proper subvariety of $X$ which is preserved by $\operatorname{Aut}_{o}(X)$. Proposition 6.3 implies the following.

Corollary 6.4. Let $X$ be a quasi-homogeneous Fano manifold of Picard number one with the boundary $E \subset X$. Then any surjective endomorphism $f: X \rightarrow X$ satisfies $f^{-1}(E)=E$ and $\left.f\right|_{X \backslash E}$ is étale.

Proof of Theorem 1.4. Assume that $X$ is a quasi-homogeneous Fano manifold of Picard number one different from the projective space. Theorem 1.4 claims that any surjective endomorphism $f: X \rightarrow X$ is bijective. To prove this, we use Corollary 6.4 to conclude that $f$ must be étale on the complement of the boundary $E$. Thus if $E$ has codimension $\geq 2$ in $X$, then $f$ is étale and must 
be bijective because a Fano manifold is simply connected. If $E$ has components of codimension 1, then by Corollary 6.4 and the purity of branch locus, those components form a divisor $D \subset X$ with $f^{-1}(D)=D$ such that $f$ is étale outside $D$. Thus $f$ is étale outside a completely invariant divisor. By Theorem 1.2, we conclude that $f$ is bijective.

Let us finish with a final remark. As noted in [HM04, p. 62], the variety of minimal rational tangents is not linear for homogeneous spaces of Picard number one, excepting the projective space. However, it is linear for some quasi-homogeneous Fano manifolds of Picard number one (e.g. [HM03, Corollary 2]). So it is essential to use Theorem 1.3 for the proof of Theorem 1.4.

\section{REFERENCES}

[Be] Beauville, A.: Endomorphisms of hypersurfaces and other manifolds, Intern. Math. Res. Notices 2001 no. 1, 53-58.

[Ho] Horikawa, E.: On deformations of holomorphic maps I, J. Math. Soc. Japan 25 (1973), $372-396$.

[Hw] Hwang, J.-M.: Deformation of holomorphic maps onto Fano manifolds of second and fourth Betti numbers 1, Ann. Inst. Fourier 57 (2007), 815-823.

[HM03] Hwang, J.-M. and Mok, N.: Finite morphisms onto Fano manifolds of Picard number 1 which have rational curves with trivial normal bundles, J. Alg. Geom. 12 (2003), 627-651.

[HM04] Hwang, J.-M. and Mok, N.: Birationality of the tangent map for minimal rational curves, Asian J. Math. 8, Special issue dedicated to Yum-Tong Siu, (2004), 51-64.

[KO] Kobayashi, S. and Ochiai, T.: Characterizations of complex projective spaces and hyperquadrics, J. Math. Kyoto Univ. 13 (1973), 31-47.

[Kr] Kollár, J.: Rational curves on algebraic varieties, Erg. d. Math. 3 Folge 32, Springer Verlag 1996.

[Na] Nakayama, N.: Zariski-decomposition and abundance, MSJ Memoirs 14, Math. Soc. Japan 2004.

[NZ] Nakayama, N. and Zhang, D.-Q.: Polarized endomorphisms of complex normal varieties, preprint RIMS-1613, Kyoto Univ. 2007.

[PS] Paranjape, K. H. and Srinivas, V.: Self maps of homogeneous spaces, Invent. Math. 98 (1989), 425-444.

[Pq] Pasquier, B.: On some smooth projective two-orbit varieties with Picard number 1, Math. Ann. 344 (2009), 963-987

Jun-Muk Hwang

Korea Institute for Advanced Study 
Seoul 130-722 Korea

E-mail: jmhwang@kias.re.kr

\section{Noboru Nakayama}

Research Institute for Mathematical Sciences

Kyoto University, Kyoto 606-8502 Japan

E-mail: nakayama@kurims.kyoto-u.ac.jp 